\title{
The $\mathrm{NH}_{2} \mathrm{D} / \mathrm{NH}_{3}$ ratio toward pre-protostellar cores around the UCH II region in IRAS 20293+3952^
}

\author{
G. Busquet ${ }^{1}$, A. Palau ${ }^{2}$, R. Estalella ${ }^{1}$, J. M. Girart ${ }^{2}$, Á. Sánchez-Monge ${ }^{1}$, S. Viti ${ }^{3}$, P. T. P. Ho ${ }^{4,5}$, and Q. Zhang ${ }^{4}$ \\ 1 Departament d'Astronomia i Meteorologia (IEEC-UB), Institut de Ciències del Cosmos, Universitat de Barcelona, \\ Martí i Franquès 1, 08028 Barcelona, Spain \\ e-mail: gbusquet@am.ub.es \\ 2 Institut de Ciències de l'Espai (CSIC-IEEC), Campus UAB, Facultat de Ciències, Torre C-5 parell, 08193 Bellaterra, Spain \\ 3 Department of Physics and Astronomy, University College London, Grower Street, London WC1E 6BT, UK \\ ${ }^{4}$ Harvard-Smithsonian Center for Astrophysics, Cambridge, MA, 02138, USA \\ 5 Academia Sinica Institute of Astronomy and Astrophysics, Taipei, Taiwan
}

Received 26 April 2010 / Accepted 16 June 2010

\begin{abstract}
Context. The deuterium fractionation, $D_{\text {frac }}$, has been proposed as an evolutionary indicator in pre-protostellar and protostellar cores of low-mass star-forming regions.

Aims. We investigate $D_{\text {frac }}$, with high angular resolution, in the cluster environment surrounding the UCH II region IRAS $20293+3952$. Methods. We performed high angular resolution observations with the IRAM Plateau de Bure Interferometer (PdBI) of the ortho$\mathrm{NH}_{2} \mathrm{D} 1_{11}-1_{01}$ line at $85.926 \mathrm{GHz}$ and compared them with previously reported $\mathrm{VLA} \mathrm{NH} \mathrm{N}_{3}$ data.

Results. We detected strong $\mathrm{NH}_{2} \mathrm{D}$ emission toward the pre-protostellar cores identified in $\mathrm{NH}_{3}$ and dust emission, all located in the vicinity of the UCH II region IRAS $20293+3952$. We found high values of $D_{\text {frac }} \simeq 0.1-0.8$ in all the pre-protostellar cores and low values, $D_{\text {frac }}<0.1$, associated with young stellar objects.

Conclusions. The high values of $D_{\text {frac }}$ in pre-protostellar cores could be indicative of evolution, although outflow interactions and UV radiation could also play a role.
\end{abstract}

Key words. stars: formation - ISM: individual objects: IRAS 20293+3952 - ISM: clouds - ISM: molecules

\section{Introduction}

Characterizing the different evolutionary stages before and after the formation of a star is crucial for understanding the process of star formation itself. Dense cores, where stars are born, are mainly studied through molecular emission of dense gas tracers, and N-bearing molecules are widely used because they do not freeze out onto dust grains until very high densities are reached $\left(\sim 10^{6} \mathrm{~cm}^{-3}\right.$, Flower et al. 2006). Several column density ratios of molecules tracing dense cores have been proposed as good chemical clocks, such as $\mathrm{NH}_{3} / \mathrm{N}_{2} \mathrm{H}^{+}$or $\mathrm{CN} / \mathrm{N}_{2} \mathrm{H}^{+}$(Hotzel et al. 2004; Fuente et al. 2005). Among these, the ratio of a deuterated species over its counterpart containing $\mathrm{H}$, i.e., the deuterium fractionation $D_{\text {frac }}$, has been found to be a good tracer of the evolutionary stage of dense cores. Both observations and models strongly suggest that in cold $(T<20 \mathrm{~K})$ and dense cores $\left(n \simeq 10^{6} \mathrm{~cm}^{-3}\right)$ C-bearing molecules are expected to deplete onto dust grains, leading to an enhancement of the deuterium fractionation (e.g., Roberts \& Millar 2000; Bacmann et al. 2003; Pillai et al. 2007) because the $\mathrm{H}_{2} \mathrm{D}^{+}$ion, the progenitor of most of the deuterated species, including $\mathrm{NH}_{2} \mathrm{D}$, is not destroyed by CO.

In particular, in low-mass star-forming regions, $D_{\text {frac }}$ is found to increase until the onset of star formation and to decrease

^ Based on observations carried out with the IRAM Plateau de Bure Interferometer. IRAM is supported by INSU/CNRS (France), MPG (Germany), and IGN (Spain). afterwards (Crapsi et al. 2005; Emprechtinger et al. 2009). In the high-mass regime, Chen et al. (2010) performed single-dish observations toward massive dense cores and find a decreasing trend of $D_{\text {frac }}$ with the evolutionary stage during the protostellar phase. However, since massive star formation takes place in cluster environments, to study $D_{\text {frac }}$ one needs to carry out observations with high angular resolution in order to separate the emission from each individual core. So far, interferometric observations of deuterated species in massive star-forming regions have only been reported by Fontani et al. (2008) and Sandell \& Wright (2010), but only the work of Fontani et al. (2008) evaluates $D_{\text {frac }}$ in the region, where they find two dense cores with $D_{\text {frac }} \simeq 0.1$.

In this Letter we present high angular resolution observations of the ortho- $\mathrm{NH}_{2} \mathrm{D} 1_{11}-1_{01}$ line at $85.926 \mathrm{GHz}$ carried out with the Plateau de Bure Interferometer toward the high-mass star-forming region IRAS $20293+3952$, located at $2 \mathrm{kpc}$ of distance (Beuther et al. 2004b) and with a luminosity of $6300 \mathrm{~L}_{\odot}$. The region is associated with an UCH II region at the border of a cloud of dense gas mapped in $\mathrm{NH}_{3}$ with high angular resolution (Palau et al. 2007), and it harbors a rich variety of young stellar objects (YSOs) and dense cores at different evolutionary stages, being thus an excellent target to study $D_{\text {frac }}$. While the northern side of the main cloud of the region contains several YSOs driving at least four molecular outflows (Beuther et al. 2004a; Palau et al. 2007), the southern side is mainly populated with starless core candidates (BIMA 3 and BIMA 4, two faint 


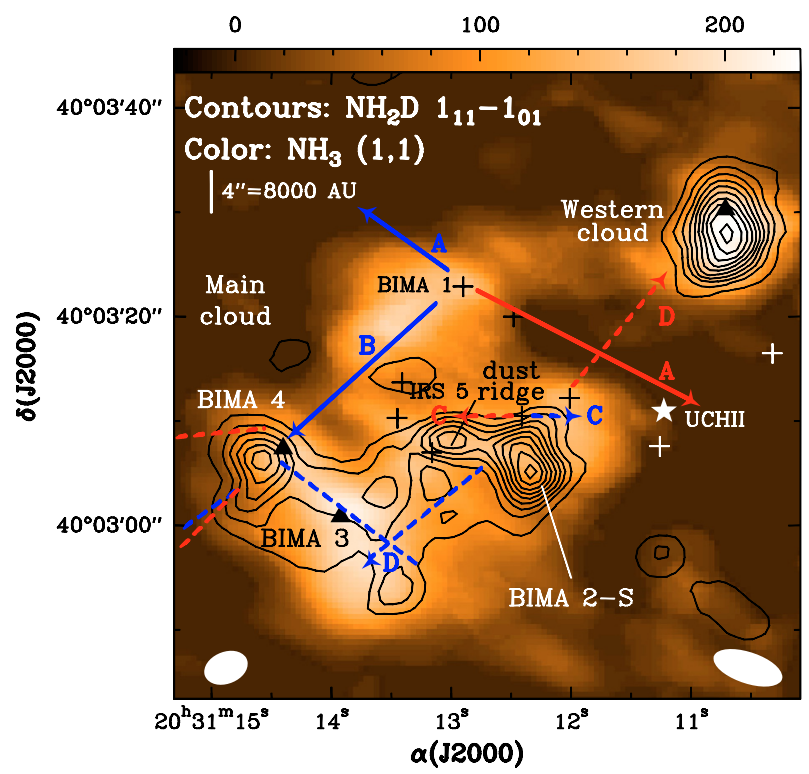

Fig. 1. Contours: $\mathrm{NH}_{2} \mathrm{D}$ zero-order moment. Contours start at $6 \%$, increasing in steps of $10 \%$ of the peak intensity, $0.58 \mathrm{Jy} \mathrm{beam}^{-1} \mathrm{~km} \mathrm{~s}^{-1}$. Color scale: $\mathrm{NH}_{3}(1,1)$ zero-order moment (Palau et al. 2007). The $\mathrm{NH}_{2} \mathrm{D}$ synthesized beam, $4^{\prime \prime} .32 \times 33^{\prime \prime} .05\left(\mathrm{PA}=111^{\circ}\right)$, and the $\mathrm{NH}_{3}$ synthesized beam are shown in the bottom left and bottom right corners, respectively. White star: position of the UCH II region (IRAS 20293+3952). Plus signs: YSOs embedded in the main cloud. Black triangles: $\mathrm{NH}_{3}$ column density peaks in the starless cores BIMA 3, BIMA 4, and the western cloud. Main cores of $\mathrm{NH}_{2} \mathrm{D}$ are labeled as western cloud, dust ridge, BIMA 2-S, and BIMA 4. The blue and red continuum/dashed arrows mark the direction of powerful/less powerful molecular outflows, respectively (Beuther et al. 2004a). Outflow B is deflected by BIMA 4 (Palau et al. 2007) and the dashed lines indicate the high-velocity gas around BIMA 4.

millimetercondensations). In addition, there is a small cloud to the northwest, the western cloud, which seems to harbor a very compact starless core (see Fig. 1 for an overview of the region). The current $\mathrm{NH}_{2} \mathrm{D}$ observations allowed us to estimate for the first time $D_{\text {frac }}$ from $\mathrm{NH}_{2} \mathrm{D} / \mathrm{NH}_{3}$ with high angular resolution toward a massive star-forming region containing dense cores and YSOs at different evolutionary stages.

\section{Observations}

The IRAM Plateau de Bure Interferometer (PdBI) was used to observe the $\mathrm{NH}_{2} \mathrm{D} 1_{11}-1_{01}$ molecular transition at $85.926 \mathrm{GHz}$ toward IRAS $20293+3952$. The observations were carried out during 2008 June 16 and December 4, with the array in the D (4 antennas) and C (6 antennas) configurations, respectively, providing projected baselines ranging from $17.5 \mathrm{~m}$ to $175 \mathrm{~m}$. The phase center was $\alpha(\mathrm{J} 2000)=20^{\mathrm{h}} 31^{\mathrm{m}} 12^{\mathrm{s}} .7, \delta(\mathrm{J} 2000)=$ $+40^{\circ} 03^{\prime} 13^{\prime \prime}$. 4 . The typical system temperatures for the receivers at $3 \mathrm{~mm}$ were $\sim 150-300 \mathrm{~K}$ during June 16 , and $\sim 100-150 \mathrm{~K}$ during December 4. Atmospheric phase correction was applied.

The $3 \mathrm{~mm}$ receiver was tuned at $86.75433 \mathrm{GHz}$ in the lower sideband. The spectral setup included other molecular transitions, which will be presented in a subsequent paper (Busquet et al., in prep.). For the ortho- $\mathrm{NH}_{2} \mathrm{D}$, we used a correlator unit of $20 \mathrm{MHz}$ of bandwidth and 512 spectral channels, which provides a spectral resolution of $\sim 0.039 \mathrm{MHz}\left(\sim 0.14 \mathrm{~km} \mathrm{~s}^{-1}\right)$. The $F W H M$ of the primary beam at $3 \mathrm{~mm}$ was $\sim 56^{\prime \prime}$. Bandpass calibration was performed by observing the quasars 3C 454.3 on June 16 and 3C 273 on December 4. Amplitude and phase calibrations were achieved by monitoring MWC 349 and $2005+403$ for both days. The phase rms was $\sim 20^{\circ}$. The absolute flux density scale was determined from MWC 349, with an estimated uncertainty $\sim 10 \%$. The data were calibrated with the program CLIC and imaged with MAPPING, and both are part of the GILDAS software package. Imaging was performed with natural weighting, obtaining a synthesized beam of 4 ". $32 \times 3$.'.05 with $\mathrm{PA}=111^{\circ}$, and rms of $20.7 \mathrm{mJy} \mathrm{beam}^{-1}$ per channel.

\section{Results}

In Fig. 1 we present the $\mathrm{NH}_{2} \mathrm{D} 1_{11}-1_{01}$ zero-order map integrated for the 6 hyperfine transitions (see Olberg et al. 1985, for a description of the transition) overlaid onto the $\mathrm{NH}_{3}(1,1)$ emission from Palau et al. (2007). The integrated $\mathrm{NH}_{2} \mathrm{D}$ emission toward the western cloud presents a compact morphology with a deconvolved size of 6". $5 \times 3$ ". $6(\sim 0.06 \mathrm{pc})$. We also found $\mathrm{NH}_{2} \mathrm{D}$ emission associated with the southern side of the main cloud, covering a spatial extension in the east-west direction of $\sim 33^{\prime \prime}$ $(0.33 \mathrm{pc})$. The emission consists of three main cores, one clearly associated with BIMA 4, another $\mathrm{NH}_{2} \mathrm{D}$ core coincident with the dust ridge, and a third core located $\sim 7^{\prime \prime}$ south of BIMA 2 (hereafter BIMA 2-S). In addition, we detected faint $\mathrm{NH}_{2} \mathrm{D}$ emission associated with BIMA 3. It is worth noting that $\mathrm{NH}_{2} \mathrm{D}$ is not (or marginally) detected on the northern side of the main cloud. In this Letter we follow the nomenclature for the YSOs and starless core candidates of Palau et al. (2007).

We studied the deuterium fractionation of the region by first computing the column density map of $\mathrm{NH}_{2} \mathrm{D}$ and then the $N\left(\mathrm{NH}_{2} \mathrm{D}\right) / N\left(\mathrm{NH}_{3}\right)$ column density ratio. For this, we extracted the spectra for positions on a grid of $2^{\prime \prime} \times 2^{\prime \prime}$, and adopted the hyperfine frequencies listed in Tiné et al. (2000). The column densities are only reported for spectra with a peak intensity greater than $5 \sigma$, and with all the hyperfine components detected.

Assuming that all levels are populated according to the same excitation temperature, $T_{\mathrm{ex}}$, the column density of the asymmetric top molecule $\mathrm{NH}_{2} \mathrm{D}$ is given by

$$
\begin{aligned}
{\left[\frac{N\left(\mathrm{NH}_{2} \mathrm{D}\right)}{\mathrm{cm}^{-2}}\right]=} & 1.94 \times 10^{3}\left[\frac{v}{\mathrm{GHz}}\right]^{2}\left[\frac{A_{\mathrm{ul}}}{\mathrm{s}^{-1}}\right]^{-1} \frac{Q\left(T_{\mathrm{ex}}\right)}{g_{\mathrm{u}}} \\
& \times \exp \left(\left[\frac{E_{\mathrm{u}}}{\mathrm{K}}\right]\left[\frac{T_{\mathrm{ex}}}{\mathrm{K}}\right]^{-1}\right) J_{v}\left(T_{\mathrm{ex}}\right) \tau_{\mathrm{m}}\left[\frac{\Delta v}{\mathrm{~km} \mathrm{~s}^{-1}}\right],
\end{aligned}
$$

where $\Delta v$ is the linewidth, $\tau_{\mathrm{m}}$ the optical depth of the main line $\left(1_{11}-1_{01}, F=2-2\right), v$ the frequency of the transition $(v=85.926 \mathrm{GHz}), E_{\mathrm{u}}$ the energy of the upper level $\left(E_{\mathrm{u}}=\right.$ $20.68 \mathrm{~K}), A_{\mathrm{ul}}=5.8637 \times 10^{-6} \mathrm{~s}^{-1}$ is the Einstein coefficient, and $J_{v}\left(T_{\mathrm{ex}}\right)=(h v / k) /\left(\mathrm{e}^{h v / k T_{\mathrm{ex}}}-1\right)$, where $h$ and $k$ are the Planck and Boltzmann constants, respectively. From the Cologne Database for Molecular Spectroscopy (CDMS, Müller et al. 2001), the upper level degeneracy is $g_{\mathrm{u}}=15$, and the partition function $Q\left(T_{\mathrm{ex}}\right)$ is estimated as $Q\left(T_{\mathrm{ex}}\right)=\alpha+\beta T_{\mathrm{ex}}{ }^{3 / 2}$, where $\alpha=3.899$ and $\beta=0.751$ are the best-fit parameters from a fit to the partition function at the different temperatures given in CDMS. The standard ortho/para ratio of 3 (e.g., Walmsley et al. 1987; Tiné et al. 2000) is already included in the partition function.

Figure 2 shows a map of the linewidth $(\Delta v)$ derived from the hyperfine fit and the total column density $N\left(\mathrm{NH}_{2} \mathrm{D}\right)$ toward the southern side of the main cloud (i.e., dust ridge core, BIMA 2-S, BIMA 3, and BIMA 4, hereafter referred to as the main cloud; see left panels) and the western cloud (right panels). The properties of the western cloud and the main cloud are somewhat different. The total optical depth $\left(\tau_{1_{11}-1_{01}}=2 \tau_{\mathrm{m}}\right)$ in the western cloud is in the range $\sim 4-15$, with a typical uncertainty of 

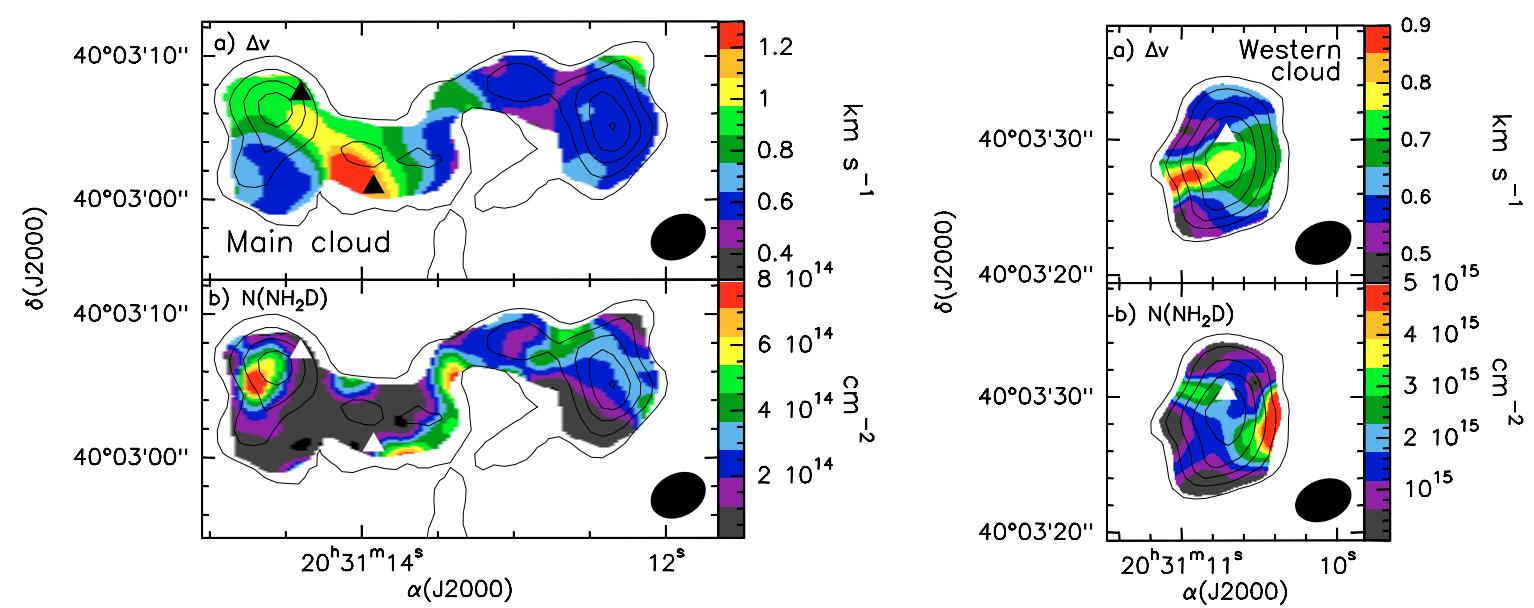

Fig. 2. Color-scale maps of linewidth and $N\left(\mathrm{NH}_{2} \mathrm{D}\right.$ ) overlaid on the $\mathrm{NH}_{2} \mathrm{D} 1_{11}-1_{01}$ integrated emission (contours) for the main cloud (left panels) and the western cloud (right panels). Triangles mark the $\mathrm{NH}_{3}$ column density peaks of BIMA 3, BIMA 4 (left), and the western cloud (right). In all panels the synthesized beam is shown in the bottom right corner.

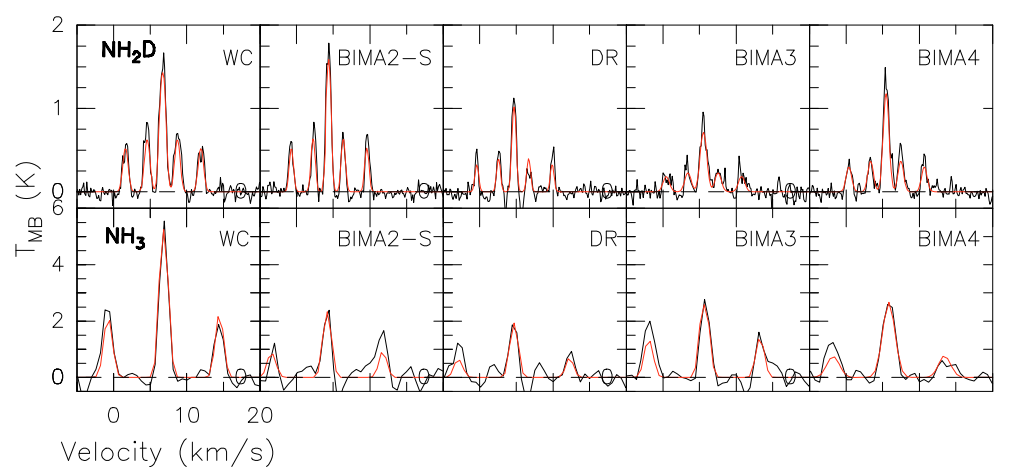

Fig. 3. Spectra (black line) toward 5 positions of the IRAS $20293+3952$ for $\mathrm{NH}_{2} \mathrm{D} 1_{11}-1_{01}$ (top) and $\mathrm{NH}_{3}(1,1)$ (bottom). The 5 positions, which are labeled in the top right corner of each panel, are, from left to right WC (western cloud), BIMA 2-S, DR (dust ridge core), BIMA 3 , and BIMA 4. The red lines show the fit to the hyperfine structure obtained as illustrated in Sect. 3.

$\sim 1.1$, whereas the total optical depth is lower toward the main cloud, between 0.5 and 4 , with a typical uncertainty of $\sim 0.5$. Concerning the linewidth in these clouds (see Fig. 2a), we find line broadening $\left(\Delta v \simeq 0.75-0.9 \mathrm{~km} \mathrm{~s}^{-1}\right)$ associated with the western cloud. Toward BIMA 3 and BIMA 4, we also find high values for the linewidth, around $\sim 0.8-1.2 \mathrm{~km} \mathrm{~s}^{-1}$, while the dust ridge core and BIMA 2-S appear to be more quiescent, with linewidths of $\sim 0.5-0.7 \mathrm{~km} \mathrm{~s}^{-1}$. Since it has been suggested that one of the outflows of the region, outflow $\mathrm{B}$, is interacting with BIMA 4 (Palau et al. 2007), this could produce the line broadening seen in $\mathrm{NH}_{2} \mathrm{D}$ in BIMA 3 and BIMA 4. Additionally, the line broadening found in the western cloud is spatially coincident (in projection) with a high-velocity feature of outflow D (Beuther et al. 2004a). Thus, the broad lines seen toward the western cloud, BIMA 3, and BIMA 4, suggest that the deuterated gas is being perturbed by the passage of the outflows (see Fig. 1).

The $\mathrm{NH}_{2} \mathrm{D}$ column density, corrected for the primary beam response, also presents significant differences between the western cloud and the main cloud. While the column density is, on average, $N\left(\mathrm{NH}_{2} \mathrm{D}\right) \simeq 25 \times 10^{14} \mathrm{~cm}^{-2}$ in the western cloud, in the main cloud the column density is slightly lower, in the range $1-8 \times 10^{14} \mathrm{~cm}^{-2}$, reaching its maximum value of $8 \times 10^{14} \mathrm{~cm}^{-2}$ close to the peak position of BIMA 4 (see Fig. 2b). The uncertainty of the $\mathrm{NH}_{2} \mathrm{D}$ column density is $\sim 25-35 \%$.

In order to properly estimate the deuterium fractionation, defined as $D_{\text {frac }}=N\left(\mathrm{NH}_{2} \mathrm{D}\right) / N\left(\mathrm{NH}_{3}\right)$, we made the $\mathrm{NH}_{3}$ VLA images using the same $u v$ range as the PdBI data $(5-50 \mathrm{k} \lambda)$, estimating the column densities for both $\mathrm{NH}_{3}$ and $\mathrm{NH}_{2} \mathrm{D}$ for the same angular scales. Finally, we convolved the $\mathrm{NH}_{2} \mathrm{D}$ and $\mathrm{NH}_{3}$ emission to a circular beam of $7 "$ (the major axis of the $\mathrm{NH}_{3}$ beam). In Fig. 3 we present the spectra obtained at the $\mathrm{NH}_{2} \mathrm{D}$ emission peak of each condensation (i.e., western cloud, BIMA 2-S, dust ridge core, BIMA 3, and BIMA 4) together with the hyperfine fit obtained toward these positions. In Table 1 we list the excitation temperature, $T_{\mathrm{ex}}$, the rotational temperature, $T_{\text {rot }}$, the $\mathrm{NH}_{2} \mathrm{D}$ and $\mathrm{NH}_{3}$ column densities, $D_{\text {frac }}$, and the $\mathrm{NH}_{3} / \mathrm{N}_{2} \mathrm{H}^{+}$ratio for each core and a few YSOs. Toward the YSOs BIMA 1 and IRS 5, we report on upper limits, with $D_{\text {frac }}<0.1$, and we cannot draw any conclusion for the behavior of $D_{\text {frac }}$ in the protostellar phase. More interestingly, in the western cloud $D_{\text {frac }}$ is $\sim 0.8$, which is the highest value of $D_{\text {frac }}$ in the region and among the highest reported in the literature (e.g., Crapsi et al. 2007; Pillai et al. 2007; Fontani et al. 2008). In the main cloud, $D_{\text {frac }}$ presents significant variations among the different cores, with $D_{\text {frac }}$ decreasing from the northwest ( $D_{\text {frac }} \simeq 0.5$ in BIMA 2 -S and the dust ridge core) to the southeast ( $D_{\text {frac }} \simeq 0.1$ in BIMA 3 and BIMA 4). This suggests a chemical differentiation along the main cloud.

\section{Discussion and summary}

Our high angular resolution study of the $\mathrm{NH}_{2} \mathrm{D}$ toward the massive star-forming region IRAS 20293+3952 reveals strong 
A\&A 517, L6 (2010)

Table 1. Summary of the main physical properties of selected cores in IRAS $20293+3952$.

\begin{tabular}{|c|c|c|c|c|c|c|c|c|c|c|}
\hline Core & $\begin{array}{c}\alpha(\mathrm{J} 2000) \\
(\mathrm{h} \mathrm{m} \mathrm{s})\end{array}$ & $\begin{array}{c}\delta(\mathrm{J} 2000) \\
\left({ }^{\circ},{ }^{\prime \prime}\right)\end{array}$ & $\begin{array}{r}T_{\text {ex }}{ }^{a} \\
(\mathrm{~K}) \\
\end{array}$ & $\tau_{\mathrm{m}}^{b}$ & $\begin{array}{l}T_{\text {rot }}{ }^{c} \\
(\mathrm{~K})\end{array}$ & $\begin{array}{c}N\left(\mathrm{NH}_{2} \mathrm{D}\right)^{d} \\
\left(\times 10^{14} \mathrm{~cm}^{-2}\right)\end{array}$ & $\begin{array}{c}N\left(\mathrm{NH}_{3}\right)^{d} \\
\left(\times 10^{14} \mathrm{~cm}^{-2}\right)\end{array}$ & $D_{\text {frac }}{ }^{e}$ & $\begin{array}{c}\mathrm{NH}_{3} / \\
\mathrm{N}_{2} \mathrm{H}^{+c}\end{array}$ & $\begin{array}{l}\text { Evol. } \\
\text { stage }^{f}\end{array}$ \\
\hline Western cloud & $20: 31: 10.70$ & $40: 03: 28.2$ & $4.8 \pm 0.2$ & 1.5 & $15 \pm 3$ & $10 \pm 2$ & $14 \pm \quad 2$ & $0.8 \pm 0.2$ & 300 & $\overline{\mathrm{PPC}}$ \\
\hline BIMA 2-S & $20: 31: 12.34$ & 40:03:05.7 & $5.4 \pm 0.3$ & 1.1 & $19 \pm 3$ & $2.8 \pm 0.6$ & $6.7 \pm 0.8$ & $0.4 \pm 0.1$ & 90 & PPC \\
\hline Dust ridge & $20: 31: 12.98$ & 40:03:08.5 & $4.6 \pm 0.3$ & 1.0 & $16 \pm 3$ & $2.8 \pm 0.7$ & $4.9 \pm 0.9$ & $0.6 \pm 0.2$ & 90 & PPC \\
\hline BIMA 3 & 20:31:13.92 & 40:03:00.9 & $5.4 \pm 1.0$ & 0.4 & $14 \pm 2$ & $1.9 \pm 0.5$ & $19 \pm 3$ & $0.10 \pm 0.04$ & 300 & PPC \\
\hline BIMA 4 & 20:31:14.56 & 40:03:06.8 & $7.5 \pm 1.6$ & 0.3 & $14 \pm 2$ & $1.2 \pm 0.4$ & $7.4 \pm 0.8$ & $0.16 \pm 0.05$ & 200 & PPC \\
\hline BIMA 1 & 20:31:12.77 & 40:03:22.6 & $\ldots$ & $\ldots$ & $24 \pm 3$ & $<1.1^{g}$ & $19 \pm 3$ & $<0.06$ & 50 & $\mathrm{O}$, no IR \\
\hline IRS 5 & $20: 31: 13.41$ & 40:03:13.7 & $\ldots$ & $\ldots$ & $24 \pm 3$ & $<1.5^{g}$ & $11 \pm 2$ & $<0.1$ & 50 & IR \\
\hline
\end{tabular}

Notes. ${ }^{(a)} T_{\mathrm{ex}}$ derived from the output parameters of the $\mathrm{NH}_{2} \mathrm{D} 1_{11}-1_{01}$ hyperfine fit. ${ }^{(b)}$ Derived from the fits to the hyperfine structure of $\mathrm{NH}_{2} \mathrm{D} 1_{11}-1_{01}$. ${ }^{(c)}$ Obtained from Palau et al. (2007). ${ }^{(d)}$ Column densities derived using the same $u v$ range and convolving the images to a circular beam of $7^{\prime \prime} .{ }^{(e)} D_{\text {frac }}=N\left(\mathrm{NH}_{2} \mathrm{D}\right) / N\left(\mathrm{NH}_{3}\right) .{ }^{(f)}$ PPC: pre-protostellar core; O: molecular outflow. ${ }^{(g)} 3 \sigma$ upper limit estimated adopting $\Delta v$ from $\mathrm{NH}_{3}$ and $T_{\mathrm{ex}}=5 \mathrm{~K}$.

$\mathrm{NH}_{2} \mathrm{D}$ emission toward starless cores, whereas $\mathrm{NH}_{2} \mathrm{D}$ is not (or marginally) detected in cores containing YSOs, which suggests that the production of $\mathrm{NH}_{2} \mathrm{D}$ is more effective in the preprotostellar phase than in the protostellar phase. Palau et al. (2007) notice that the starless cores in this region seem to be predominant on the southern side of the main cloud and in the western cloud, while the northern side of the main cloud harbors all the YSOs known in the region, suggestive of the dense gas in the main cloud being progressively more evolved as it moves from south to north. In addition, chemical differentiation among pre-protostellar and protostellar cores was also found by Palau et al. (2007) using the $\mathrm{NH}_{3} / \mathrm{N}_{2} \mathrm{H}^{+}$ratio, which was high for pre-protstellar cores and low in protostellar cores (see Col. 9 of Table 1). Thus, for this region, the behavior of $D_{\mathrm{frac}}$, measured from $\mathrm{NH}_{2} \mathrm{D} / \mathrm{NH}_{3}$, is similar to the behavior of $\mathrm{NH}_{3} / \mathrm{N}_{2} \mathrm{H}^{+}$ratio, suggesting that both ratios can be used to distinguish between pre-protostellar and protostellar cores and that both ratios could be related with the evolutionary stage of the dense gas.

A possible interpretation of the differences in $D_{\text {frac }}$ seen in the pre-protostellar cores of region could be that they are in different evolutionary stages. According to the study of Crapsi et al. (2005), there is an increasing trend for $D_{\text {frac }}$ as the starless core approaches the onset of gravitational collapse (from 0.030.1 in the youngest cores to $0.1-0.4$ toward the most evolved cores). This would indicate that the western cloud is the most evolved pre-protostellar core and that BIMA 3 and BIMA 4 are less evolved. However, in regions of massive star formation, typically associated with clustered environments, other factors, like temperature, UV radiation, and/or molecular outflows, can play important roles in altering the chemistry, and then it is not clear whether this trend is related to the evolutionary stage of preprotostellar cores.

A comparison of $D_{\text {frac }}$ between the western cloud and BIMA 3/BIMA 4, all of them having similar temperatures but very different values of $D_{\text {frac }}$ (see Table 1 ), indicates that temperature is not an important factor in determining $D_{\text {frac }}$ for temperatures around $15 \mathrm{~K}$. Palau et al. (2007) find evidence that UV radiation from the UCH II region affects the chemistry at the western edge of the main cloud facing the UCH II region, so it could affect BIMA 2-S and the western cloud. In particular, the presence of a cavity between the UCH II region and the western cloud suggests that this cloud could be photo-illuminated by the UCH II region. However, while the western cloud and BIMA 2$\mathrm{S}$ could be locally affected by the UV radiation, this is not the case for BIMA 3 and BIMA 4, for which the high visual extinction in the main cloud prevents UV photons from penetrating. Finally, Fontani et al. (2009) point out that shocks in outflows could modify $D_{\text {frac }}$. This could be the case for the western cloud, BIMA 3, and for BIMA 4. While the interaction of an outflow with the western cloud is not evident, Palau et al. (2007) have already proposed that the powerful outflow B is interacting with BIMA 4, producing the deflection of the outflow and thus the ejection of high-velocity material in different directions. This could affect BIMA 4 and BIMA 3 because high-velocity $\mathrm{SiO}$ is seen close to these cores (see Fig. 1 and Beuther et al. 2004a). This interpretation is reinforced by the line broadening of $\mathrm{NH}_{2} \mathrm{D}$ observed towards BIMA 3 and BIMA 4 (see Fig. 2). In conclusion, while $D_{\text {frac }}$ in IRAS $20293+3952$ may be locally affected by the interaction of outflows and UV radiation, $D_{\text {frac }}$ is lower toward YSOs than toward pre-protostellar cores, with a possible evolutionary trend in the pre-protostellar phase, which deserves further study.

Acknowledgements. We thank the anonymous referee for his/her useful comments and suggestions. We acknowledge the IRAM staff for their support during the data reduction. G.B., A.P., R.E., J.M.G., and Á.S.-M. are supported by the Spanish MEC grant AYA2005-08523-C03 and the MICINN grant AYA200806189-C03 (co-funded with FEDER funds). A.P. is supported by a JAE-Doc CSIC fellowship co-funded with the European Social Fund.

\section{References}

Bacmann, A., Lefloch, B., Ceccarelli, C., et al. 2003, ApJ, 585, L55

Beuther, H., Schilke, P., \& Gueth, F. 2004a, ApJ, 608, 330

Beuther, H., Schilke, P., \& Wyrowski, F. 2004b, ApJ, 615, 832

Chen, H.-R., Liu, S.-Y., Su, Y.-N., \& Zhang, Q. 2010, ApJ, 713, L50

Crapsi A., Caselli P., Walmsley C. M., et al. 2005, ApJ, 619, 379

Crapsi, A., Caselli, P., Walmsley, C. M., \& Tafalla, M. 2007, A\&A, 470, 221

Emprechtinger, M., Caselli, P., Volgenau, N. H., Stutzki, J., \& Wiedner, M. C. 2009, A\&A, 493, 89

Flower, D. R., Pineau des Forêts, G., \& Walmsley, C. M. 2006, A\&A, 456, 215

Fontani, F., Caselli, P., Bourke, T. L., Cesaroni, R., \& Brand, J. 2008, A\&A, 477, L45

Fontani, F., Zhang, Q., Caselli, P., \& Bourke, T. L 2009, A\&A, 499, 233

Fuente, A., Rizzo, J. R., Caselli, P., Bachiller, R., \& Henkel, C. 2005, A\&A, 433, 535

Hotzel, S., Harju, J., \& Walmsley, C. M. 2004, A\&A, 415, 1065

Müller, H. S. P., Thorwirth, S., Roth, D. A., \& Winnewisser, G. 2001, A\&A, 370, L49

Olberg, M., Bester, M., Rau, G., et al. 1985, A\&A, 142, L1

Palau, A., Estalella, R., Girart, J. M., et al. 2007, A\&A, 465, 219

Pillai, T., Wyrowski, F., Hatchell, J., Gibb, A. G., \& Thompson, M. A. 2007, A\&A, 467, 207

Roberts, H., \& Millar, T. J. 2000, A\&A, 361, 388

Sandell, G., \& Wright, M. 2010, ApJ, 715, 919

Tiné, S., Roueff, E., Falgarone, E., Gerin, M., \& Pineau des Forêts, G. 2000, A\&A, 356, 1039

Walmsley, C. M., Hermsen, W., Henkel, C., Mauersberger, R., \& Wilson, T. L. 1987, A\&A, 172, 311 